\title{
A robust diagnosis method for speed sensor fault based on stator currents in the RFOC induction motor drive
}

\author{
Cuong Dinh Tran', Pavel Brandstetter², Minh Chau Huu Nguyen ${ }^{3}$, \\ Sang Dang $\mathrm{Ho}^{4}$, Bach Hoang Dinh ${ }^{5}$, Phuong Nhat Pham ${ }^{6}$ \\ ${ }^{1,4,6}$ Faculty of Electrical and Electronics Engineering, Ton Duc Thang University, Vietnam \\ ${ }^{5}$ Power System Optimization Research Group, Faculty of Electrical and Electronics Engineering, \\ Ton Duc Thang University, Vietnam \\ ${ }_{1,2,3,4,6}$ Faculty of Electrical Engineering and Computer Science, VSB-Technical University of Ostrava, Czech Republic
}

\section{Article Info}

\section{Article history:}

Received Aug 27, 2019

Revised Nov 26, 2019

Accepted Dec 10, 2019

\section{Keywords:}

Diagnosis

Fault-tolerant-control (FTC)

Induction motor (IM)

RFOC

Speed sensor

\begin{abstract}
A valid diagnosis method for the speed sensor failure (SSF) is an essential requirement to ensure the reliability of Fault-Tolerant Control (FTC) models in induction motor drive (IMD) systems. Most recent researches have focused on directly comparing the measured and estimated rotor speed signal to detect the speed sensor fault. However, using that such estimated value in both the fault diagnosis and the controller reconfiguration phases leads to the insufficient performance of FTC modes. In this paper, a novel diagnosistechnique based on the stator current model combined with a confusion prevention condition is proposed to detect the failure states of the speed sensor in the IMD systems. It helps the FTC mode to separate between the diagnosis and reconfiguration phases against a speed sensor fault. This proposed SSF diagnosis method can also effectively apply for IMs' applications at the low-speed range where the speed sensor signal often suffers from noise. MATLAB/Simulink software has been used to implement the simulations in various speed ranges. The achieved results have demonstrated the capability and effectiveness of the proposed SSF method against speed sensor faults.
\end{abstract}

Copyright (c) 2020 Institute of Advanced Engineering and Science. All rights reserved.

\section{Corresponding Author:}

Bach Hoang Dinh,

Power System Optimization Research Group,

Faculty of Electrical and Electronics Engineering,

Ton Duc Thang University,

19 Nguyen Huu Tho, District 7, Ho Chi Minh City, Vietnam.

Email: dinhhoangbach@tdtu.edu.vn

NOMENCLATURE

$\begin{array}{ll}\boldsymbol{\Psi}_{S}^{S} & \text { Stator flux vector in }[\alpha, \beta] \text { coordinate system } \\ \boldsymbol{\Psi}_{R}^{S} & \text { Rotor flux vector in }[\alpha, \beta] \text { coordinate system } \\ \boldsymbol{i}_{S}^{S} & \text { Stator current vector in }[\alpha, \beta] \text { coordinate system } \\ \boldsymbol{i}_{R}^{S} & \text { Rotor current vector in }[\alpha, \beta] \text { coordinate system } \\ \boldsymbol{u}_{S}^{S} & \text { Stator voltage vector in }[\alpha, \beta] \text { coordinate system } \\ u_{S \alpha}, u_{S \beta} & \text { Stator voltage component in }[\alpha, \beta] \text { system } \\ u_{S x}, u_{S y} & \text { Stator voltage component in }[\mathrm{x}, \mathrm{y}] \text { system }\end{array}$




$\begin{array}{ll}u_{a}, u_{b}, u_{c} & \text { Stator voltage component in }[\mathrm{a}, \mathrm{b}, \mathrm{c}] \text { system } \\ i_{S x} & \text { Flux current component } \\ i_{S y} & \text { Torque current component } \\ i_{m} & \text { Magnetizing current } \\ R_{S}, R_{R} & \text { Stator and rotor resistance } \\ L_{S}, L_{R} & \text { Stator and rotor inductance } \\ L_{m} & \text { Magnetizing inductance } \\ T_{R} & \text { Rotor time constant } \\ \omega_{m} & \text { Mechanical angular speed } \\ \omega_{e n} & \text { Mechanical angular speed of the encoder } \\ p & \text { Pole pair number } \\ \psi_{R} & \text { Nominal rotor flux } \\ \psi_{R \alpha}, \psi_{R \beta} & \text { Rotor flux component in }[\alpha, \beta] \text { system } \\ \gamma & \text { Rotor flux angle }\end{array}$

\section{INTRODUCTION}

Due to the advantages of economics, ruggedness, self-starting, and stable in operation, the threephase induction motor (IM) has become the most popular electrical machine in the industry. In the past, IMs were often applied in the uncontrollable speed applications. Nowadays, with the advanced technologies in high-performance power converters and modern control algorithms, IMs can operate more flexibly in both fixed-speed and variable-speed drives [1].

Typically, the separate controls of speed and torque are two primary requirements for all electrical drives. Various methods have been proposed to control IMs' drive systems and classified into two main groups: Scalar Control (SC) and Vector Control (VC). In SC techniques, the torque and speed are controlled based on a constant volts-per-hertz ratio principle. It is simple, and no sensors required, but it is unable to control the torque and speed of IMs at the same time [2]. On the other hand, the VC technique in which the rotor field-oriented control (RFOC) is the most typical method can overcome that problem of the SC technique. In the RFOC model, the stator-current vector is separated into two elements $i_{S x}$ and $i_{S y}$, which are perpendicular to each other. As a result, the torque and the rotor flux can be independently controlled by adjusting either $\mathrm{i}_{\mathrm{Sx}}$ or $\mathrm{i}_{\mathrm{Sy}}$, respectively. It is similar to the control principles of separately excited DC motors, where both the torque and speed can be controlled precisely at the same time in a wide speed range. Thus, this RFOC model can be applied to many complex control applications in the industry. However, to achieve high performance, RFOC needs the accurately structural parameters of IMs as well as the feedback signals from sensors. Thus, the quality of IMDs depends on not only the condition of the machines (both electrical and mechanical parts) but also the states of sensors (reliable or failure) [3].

In practice, abnormal operation of IMs could be happened due to the malfunctions of mechanical/ electrical parts or sensor errors where the speed sensor failure is one of the most severe problems [4-6]. It makes the loss or inaccuracy in feedback signals and thus leads to incorrect actions of the controller. Furthermore, if such sensor failures cannot be detected and solved quickly, it can lead to more serious problems and then causes to damage the whole IM drive system [7]. In order to limit the effect of faulty sensors in the operation of IMs, FTC methods have been developed recently to maintain the stable operation of IMs. Those methods are classified into two major groups, Active FTC and Passive FTC, where the passive techniques are often designed to work offline against some predefined failures without changing the control structure $[8,9]$.

On the contrary, the active solutions need online checking the status of sensors and reconfigure the control structure if finding any failure of the actual measurement system. The active FTC systems comprise three sequential steps: fault detection, fault isolation, and reconfiguration, where the fault detection and fault isolation can also be combined in one step called the fault diagnosis. In this paper, we only focus on the diagnosis of speed sensor fault based on the active FTC scheme.

The most common approach in the SSF diagnosis is based on the comparison between the physical sensor signals and state variables of the estimator (or observer). Any speed sensor fault will be detected if the error between physical and estimated signals exceeds a specific threshold [10-12]. According to the RFOC method, the electrical torque can be controlled by adjusting the $\mathrm{i}_{\text {Sy }}$ element in the rotating 
coordinate system [x, y]. Moreover, due to the relationship between the electrical torque and the speed of IMs, to enhance the effectiveness and stability of the fault diagnosis algorithm, FTC needs four signals including physical and estimated rotor speeds as well as the $\mathrm{i}_{\text {Sy }}$ element of the measured and estimated stator currents $[6,13]$. Another approach for diagnosing a speed sensor fault based on an adaptive rotor-resistance observer has been proposed in [5]. It was based on the idea that if there is a signal failure from the speed sensor occurring, the estimated rotor resistance is significantly changed in its amplitude. Thus, the authors analyzed the difference between the estimated and real rotor resistances to determine the SSF case.

In this paper, we propose a novel method combining two methodologies based on stator currents and based on the reference rotor speed to diagnose any failure of the speed sensor. The proposed diagnosis algorithm includes two main parts. The first, we compare the measured and estimated stator currents together to detect the possible fault in the speed sensor. Next, a difference between the measured and the reference rotor speeds is checked to prevent the confusion by the failure of current sensors themselves. It means that we could base on the difference between the measured and estimated stator currents to diagnose any possible faults in the speed sensor instead of directly checking between measured and estimated rotor speeds. This proposed SSF diagnosis method can effectively apply for IMs' applications at the low-speed range where the speed sensor signal often suffers from noise while other similar algorithms would be complicated to handle in that such condition. Besides, an estimation value of rotor-time constant (RTC) is also applied to enhance the accuracy of the proposed diagnosis method. The simulations of FTC in MATLAB/Simulink have been implemented in various speed ranges to verify the effectiveness of the proposed method. The results show that the FTC using the proposed diagnosis algorithm effectively works in both normal and low-speed ranges. The remaining of this paper is organized as follows: In Section 2, the main ideas of the proposed fault diagnosis method are described in details; in Section 3, the performance of the proposed approach is verified through simulations of FTC in various operating speeds; and finally, conclusions are given in Section 4

\section{FAULT DIAGNOSIS ALGORITHM FOR THE SPEED SENSOR FAILURE}

This section includes two contents. The mathematical model based RFOC of IMs is presented in the first, and the main idea of the proposed fault diagnosis algorithm of FTC is described in detail later.

\subsection{Mathematical model of IMD according to the RFOC technique}

According to the RFOC technique, the space vector of stator currents is separated into two perpendicular elements $i_{S x}$ and $i_{S y}$ in the rotating coordinate system $[x, y]$, as shown in Figure 1 [14] where the magnetizing current corresponding to the rotor flux is standing on the axis $\mathrm{x}$. In this way, the rotor flux can be controlled by $i_{S x}$, and $i_{S y}$ is used to control the electrical torque of IMs similar to the idea of controlling separately excited DC motors.

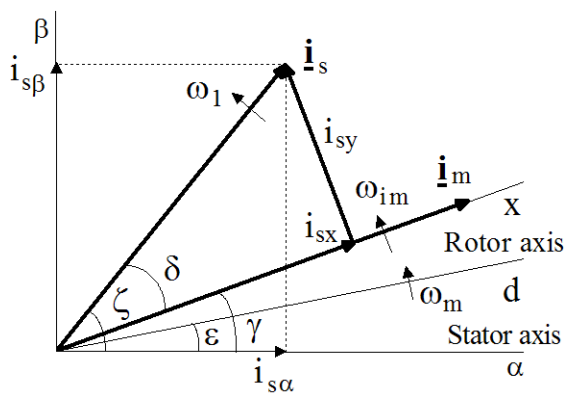

Figure 1. Principle of vector control

The three-phase stator currents and the rotor speed signal measured from sensors and the voltage signals calculated from switching signals of the inverter combining DC link voltage are transferred to the RFOC control loop, as shown in Figure 2. The Clark's formula is used to transform the quantities from the three-phase system $[a, b, c]$ to the stationary coordinate system $[\alpha, \beta]$ as shown in (1) and the Park's formula is applied to transform the stator currents from the stationary coordinate system $[\alpha, \beta]$ to the rotating coordinate system $[\mathrm{x}, \mathrm{y}]$ as shown by (2). That current model of IMs is applied to calculate the magnetizing current corresponding to the rotor flux, flux angular speed, and rotor flux angle [15]. We can see the transformations as following: 


$$
\begin{aligned}
& {\left[\begin{array}{l}
i_{S \alpha} \\
i_{S \beta}
\end{array}\right]=\left[\begin{array}{cc}
1 & 0 \\
\frac{1}{\sqrt{3}} & \frac{2}{\sqrt{3}}
\end{array}\right]\left[\begin{array}{l}
i_{S a} \\
i_{S b}
\end{array}\right]} \\
& {\left[\begin{array}{c}
i_{S x} \\
i_{S y}
\end{array}\right]=\left[\begin{array}{cc}
\cos \gamma & \sin \gamma \\
-\sin \gamma & \cos \gamma
\end{array}\right]\left[\begin{array}{l}
i_{S \alpha} \\
i_{S \beta}
\end{array}\right]}
\end{aligned}
$$

The dynamic model of IMs in the stationary system $[\alpha, \beta]$ is described as the following formulas:

$$
\left\{\begin{array}{l}
\boldsymbol{u}_{S}^{S}=R_{S} i_{S}^{S}+\frac{d \Psi_{S}^{S}}{d t} ; 0=R_{R} i_{R}^{S}+\frac{d \Psi_{R}^{S}}{d t}-j \omega_{R} \Psi_{R}^{S} \\
\Psi_{S}^{S}=L_{S} i_{S}^{S}+L_{m} i_{R}^{S} ; \Psi_{R}^{S}=L_{m} i_{S}^{S}+L_{R} i_{R}^{S}
\end{array}\right.
$$

and the electrical torque produced by the induction motor is presented by

$$
T_{e}=\frac{3}{2} \frac{p}{2} \frac{L_{m}}{L_{R}}\left(\psi_{R \alpha} i_{S \beta}-\psi_{R \beta} i_{S \alpha}\right)
$$

From the mathematical relationships described above, the control block diagram based on RFOC applied in this paper is expressed in Figure 2. The controller executes the control loops to achieve the control variables, and then these variables are transformed from the rotating reference frame back to the stationary reference frame by reversing the Park's transformation. Finally, the reverse Clark's transformation is applied to transform the control variables from the two-phase system $[\alpha, \beta]$ to a three-phase system $[\mathrm{a}, \mathrm{b}, \mathrm{c}]$ and these reference variables are employed to control the switching inverter by the sine pulse width modulation (SPWM) method. Thus, the performance of the controller based on RFOC is closely related to the accuracy of physical signals measured from the current and speed sensors, and any abnormal condition of the measurement system could affect the operation quality of IMDs.

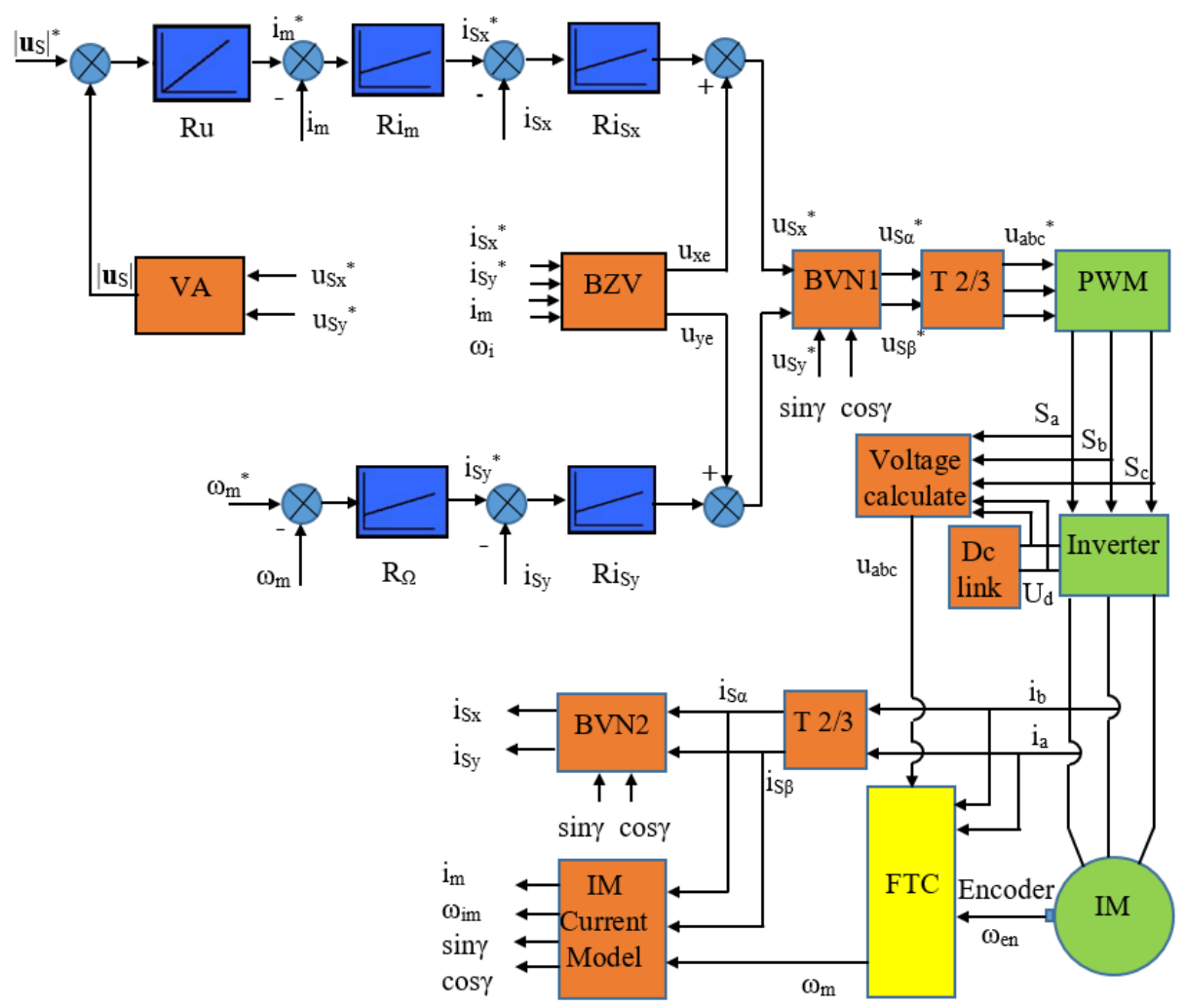

Figure 2. Control block diagram based on the RFOC combined with FTC 
In order to improve the IMDs' operation under abnormal conditions where the measurement signals are insufficient, an FTC module is added on the control schemes to diagnose and resolve the failure of sensors. Its methodology is based on comparing the physical signals from sensors and the estimated values from observers (estimators). It works like a switch between two controlling modes, normal and fault tolerate modes. At normal condition, the controller uses the measured rotor-speed signal as the feedback signal to implement the control action, but if there is any malfunction happened in the rotor-speed sensor, it is switched to FTC mode where the physical signal will be replaced by the estimated values. Thus, we need to supervise and detect any possible failure of that such speed sensor, and that is the aim of this research.

\subsection{The diagnosis algorithm to detect failures in the speed sensor}

As mentioned in the previous part, the rotor speed signal from the sensor has an important role in determining the state variables of IMDs, like the rotor flux, electromagnetic torque, etc. in the control scheme. Therefore, if occurring any failures of the speed sensor during operation, we need to detect, isolate, and replace that insufficient signal by a more suitable signal immediately. In the past, most of the existing SSF diagnosis methods [10-12] directly detect the speed sensor failure according to the deviation between the measured speed signal and the estimated signal. The below condition is a fault detector where a fault of the speed sensor can be decided if the error exceeds a specific threshold [11] as shown by

$$
\begin{aligned}
& \mathrm{F}_{\text {speed }}=\left|\omega_{R_{-} m}-\omega_{R_{-} \_s t}\right| \geq \text { threshold } ; \\
& \bullet \text { threshold }=\left\{\begin{array}{l}
0.045 *\left|\omega_{\mathrm{R}_{-} \text {ref }}\right| ; \text { if rotor }- \text { speed }>200 \mathrm{rpm} \\
0.1 *\left|\omega_{\mathrm{R} \_ \text {ref }}\right| ; \text { if rotor }- \text { speed } \leq 200 \mathrm{rpm}
\end{array}\right.
\end{aligned}
$$

Consequently, the estimated speed signal is used in both the diagnosis phase and the reconfigure phase of the control scheme in the FTC mode [12]. It makes the system highly depending on the sensitivity of the speed estimation algorithm that could lead to insufficient performance when there is any bad interference in that such estimator's quality. Moreover, at the low-speed range, the speed sensor signal often suffers from noise. Thus, the result of the SSF diagnosis could be seriously affected by the accuracy of the speed measurement system. Therefore, it is necessary to develop some new ideas to enhance the performance of the FTC mode in various speed zones.

In this proposed FTC approach, we suggest the SSF diagnosis algorithm based on the current model where the comparison between the measured and estimated stator currents is applied to detect the speed sensor failure. After that, the speed signal in the controller based RFOC uses the estimated rotor speed determined from the model reference adaptive system (MRAS) [15-17] in the reconfiguration step. From the current model of IMs [18], the actual stator currents can be calculated from the rotor speed and the rotor flux in the stationary coordinate system as.

$$
i_{S}^{S}=\frac{1}{L_{m}}\left(1-j T_{R} \omega_{R}\right) \boldsymbol{\psi}_{R}^{S}+\frac{T_{R}}{L_{m}} \frac{d \boldsymbol{\psi}_{R}^{S}}{d t}
$$

On the other hand, the rotor flux variable $\Psi_{R}^{S}$ is calculated from the stator voltage, stator current, and structural parameters of IMs according to the voltage model [18]

$$
\boldsymbol{\psi}_{R}^{S}=\frac{L_{R}}{L_{m}}\left(\int\left(\boldsymbol{u}_{S}^{S}-R_{S} \boldsymbol{i}_{S}^{S}\right) d t-\left[\frac{L_{S} L_{R}-L_{m}^{2}}{L_{R}}\right] \boldsymbol{i}_{S}^{S}\right)
$$

Suppose that we replace the actual rotor speed $\omega_{R}$ with the measured signal of the speed sensor $\omega_{R} \_m$ in $(6)$, the estimated stator current can be correspondingly calculated by

$$
\hat{\boldsymbol{i}}_{S}^{S}=\frac{1}{L_{m}}\left(1-j T_{R} \omega_{R_{-} m}\right) \boldsymbol{\psi}_{R}^{S}+\frac{T_{R}}{L_{m}} \frac{d \boldsymbol{\psi}_{R}^{S}}{d t}
$$

From (6) and (8), the difference between actual and estimated stator currents can be displayed by

$$
\left(i_{S}^{S}-\hat{i}_{S}^{S}\right)=j\left(\omega_{R_{-} m}-\omega_{R}\right) \frac{T_{R}}{L_{m}} \psi_{R}^{S}
$$


From (9), by analyzing the stator current space vector and the rotor flux space vector in the stationary system $[\alpha, \beta]$, we can receive equations $(10),(11),(12)$ as follows:

$$
\begin{aligned}
& {\left[\left(i_{S \alpha}-\hat{i}_{S \alpha}\right)+j\left(i_{S \beta}-\hat{i}_{S \beta}\right)\right]=\left(\omega_{R_{-} m}-\omega_{R}\right) \frac{T_{R}}{L_{m}}\left(j \psi_{R \alpha}-\psi_{R \beta}\right)} \\
& \Rightarrow\left\{\begin{array} { l } 
{ [ ( i _ { S \alpha } - \hat { i } _ { S \alpha } ) = ( \omega _ { R _ { - } m } - \omega _ { R } ) \frac { T _ { R } } { L _ { m } } ( - \psi _ { R \beta } ) } \\
{ ( i _ { S \beta } - \hat { i } _ { S \beta } ) ] = ( \omega _ { R _ { - } m } - \omega _ { R } ) \frac { T _ { R } } { L _ { m } } ( \psi _ { R \alpha } ) }
\end{array} \Leftrightarrow \left\{\begin{array}{l}
{\left[\left(\hat{i}_{S \alpha}-i_{S \alpha}\right) \psi_{R \beta}=\left(\omega_{R_{-} m}-\omega_{R}\right) \frac{T_{R}}{L_{m}}\left(\psi_{R \beta}^{2}\right)\right.} \\
\left.\left(i_{S \beta}-\hat{i}_{S \beta}\right)\right] \psi_{R \alpha}=\left(\omega_{R_{-} m}-\omega_{R}\right) \frac{T_{R}}{L_{m}}\left(\psi_{R \alpha}^{2}\right)
\end{array}\right.\right. \\
& \Rightarrow\left(\hat{i}_{S \alpha}-i_{S \alpha}\right) \psi_{R \beta}+\left(i_{S \beta}-\hat{i}_{S \beta}\right) \psi_{R \alpha}=\left(\omega_{R_{-} m}-\omega_{R}\right) \frac{T_{R}}{L_{m}}\left(\psi_{R \alpha}^{2}+\psi_{R \beta}^{2}\right)
\end{aligned}
$$

Thus, the difference between the actual and measured rotor speed can be determined according to the stator current and the rotor flux in the stationary system $[\alpha, \beta][16]$ as below.

$$
\left(\omega_{R}-\omega_{R_{-} m}\right)=\frac{L_{m}}{T_{R} \cdot\left(\psi_{R \alpha}^{2}+\psi_{R \beta}^{2}\right)}\left[\left(i_{S \alpha}-\hat{i}_{S \alpha}\right) \cdot \psi_{R \beta}+\left(\hat{i}_{S \beta}-i_{S \beta}\right) \cdot \psi_{R \alpha}\right]
$$

Where, the actual stator currents $\mathrm{i}_{\mathrm{S} \alpha}, \mathrm{i}_{\mathrm{S} \beta}$ are measured from the current sensors and transformed into the stationary system $[\alpha, \beta]$ by Clark's formula; the estimated stator currents $\hat{i}_{S \alpha}, \hat{i}_{S \beta}$ are derived from following differential equations [19]:

$$
\begin{aligned}
& \frac{d i_{S \alpha}}{d t}=\left(\frac{L_{R}}{L_{S} L_{R}-L_{m}^{2}}\right)\left[u_{\alpha}-R_{S} i_{S \alpha}+\frac{L_{m}^{2}}{L_{R}} \omega_{R} i_{S \beta}+\frac{L_{m} R_{R}}{L_{R}} i_{R \alpha}+L_{m} \omega_{R} i_{R \beta}\right] \\
& \frac{d i_{S \beta}}{d t}=\left(\frac{L_{R}}{L_{S} L_{R}-L_{m}^{2}}\right)\left[u_{\beta}-R_{S} i_{S \beta}-\frac{L_{m}^{2}}{L_{R}} \omega_{R} i_{S \alpha}-L_{m} \omega_{R} i_{R \alpha}+\frac{L_{m} R_{R}}{L_{R}} i_{R \beta}\right] \\
& \frac{d i_{R \alpha}}{d t}=\left(\frac{L_{S} L_{m}}{L_{m}^{2}-L_{S} L_{R}}\right)\left[\frac{1}{L_{S}} u_{\alpha}-\frac{R_{S}}{L_{S}} i_{S \alpha}+\omega_{R} i_{S \beta}+\frac{R_{R}}{L_{m}} i_{R \alpha}+\frac{L_{R}}{L_{m}} \omega_{R} i_{R \beta}\right] \\
& \frac{d i_{R \beta}}{d t}=\left(\frac{L_{S} L_{m}}{L_{m}^{2}-L_{S} L_{R}}\right)\left[\frac{1}{L_{S}} u_{\beta}-\omega_{R} i_{S \alpha}-\frac{R_{S}}{L_{S}} i_{S \beta}-\frac{L_{R}}{L_{m}} \omega_{R} i_{R \alpha}+\frac{R_{R}}{L_{m}} i_{R \beta}\right]
\end{aligned}
$$

Moreover, in (13) the rotor time coefficient (RTC), $T_{R}=R_{R} / L_{R}$, is an important parameter that affects the accuracy of the diagnosis algorithm. Due to the influence of the environment temperature in operation, $\mathrm{T}_{\mathrm{R}}$ can be changed, but we cannot directly measure this quantity [20-25]. Thus, we need to estimate the appropriate rotor time coefficient because it could cause an undesired error in the diagnosis method if it is far from the actual value. From the mathematical model of IMs, we have a relationship of the rotor time coefficient, magnetizing current, magnetizing inductance, and flux component current as described by

$$
\begin{aligned}
& T_{R} \frac{d\left|\vec{i}_{m}\right|}{d t}+\left|\vec{i}_{m}\right|=i_{S x} \\
& \psi_{R}=L_{m}\left|\vec{i}_{m}\right|
\end{aligned}
$$

From two above equations, we estimate the rotor time coefficient from

$$
\hat{T}_{R}=\frac{1}{\psi_{R}} \int\left(L_{m} i_{S x}-\psi_{R}\right) d t
$$



rewritten by

By substituting (20) into (13), the difference between the actual and measured rotor speed can be

$$
\mathrm{F}_{\text {indication }}=\left(\omega_{R}-\omega_{R_{-} m}\right)=\frac{L_{m}}{\hat{T}_{R} \cdot\left(\psi_{R \alpha}^{2}+\psi_{R \beta}^{2}\right)}\left[\left(i_{S \alpha}-\hat{i}_{S \alpha}\right) \cdot \psi_{R \beta}+\left(\hat{i}_{S \beta}-i_{S \beta}\right) \cdot \psi_{R \alpha}\right]
$$

It is the indication function $\left(F_{\text {indication }}\right)$ to identify the speed sensor failure according to the difference between measured and estimated stator currents, the rotor flux, and the estimated rotor time coefficient. In the normal operation condition, the estimated stator currents should be the same with the measured signals from current sensors, so the value of the speed error indication function in (21) approximately equals to zero. If occurring any failure of the speed sensor, there is a significant deviation between the estimated and measured current

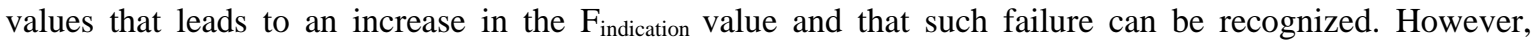
this $F_{\text {indication }}$ function could make a mistake in identifying the speed sensor fault if the current sensors fail themselves. To prevent this mistake, an additional condition called $F_{\text {ref }}$ comparing between the measured speed $\omega_{\mathrm{R}_{\_} \mathrm{m}}$ and the reference speed $\omega_{\mathrm{R}_{\_} \text {ref }}$ is added to the diagnosis algorithm as shown by

$$
\mathrm{F}_{r e f}=\left|\omega_{R_{-} m}-\omega_{R_{-}} r e f\right|
$$

Finally, the proposed diagnosis algorithm includes two parts, as described in (21), (22). The first one comparing between the measured and estimated stator currents to detect the possible fault in the speed sensor, and the second comparing between the measured and the reference rotor speeds to prevent the confusion by the possible failure of current sensors themselves. It is called the $\mathrm{F}_{\text {speed }}$ function shown by

$$
\mathrm{F}_{\text {speed }}=\left\{\begin{array}{l}
\mathrm{F}_{\text {indication }}=\frac{L_{m}}{\hat{T}_{R} \cdot\left(\psi_{R \alpha}^{2}+\psi_{R \beta}^{2}\right)}\left[\left(i_{S \alpha}-\hat{i}_{S \alpha}\right) \cdot \psi_{R \beta}+\left(\hat{i}_{S \beta}-i_{S \beta}\right) \cdot \psi_{R \alpha}\right] \geq \text { threshold_1 } \\
\mathrm{F}_{\text {ref }}=\left|\omega_{R_{-} m}-\omega_{R_{-} \text {ref }}\right| \geq \text { threshold_2 } 2
\end{array}\right.
$$

The SSF diagnosis algorithm can surely identify any speed sensor fault according to the $\mathrm{F}_{\text {speed }}$ where the values of "threshold_1" and "threshold_2" are predefined depending on the accuracy level of the actually installed sensors. In this paper, we propose the setting values about $10 \%$ of the rated stator current, and $10 \%$ of the reference rotor speed for threshold_1 and threshold_2, respectively. Thus, the proposed SSF diagnosis algorithm can be described as the flowchart in Figure 3.

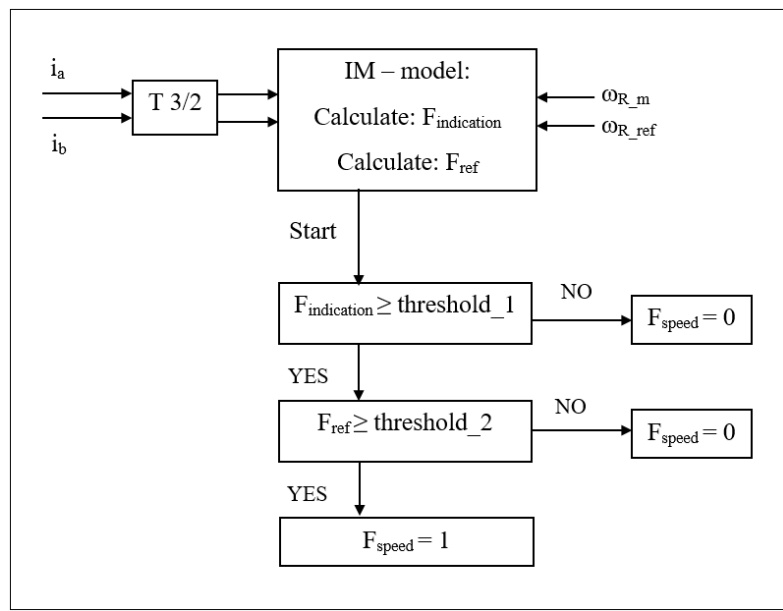

Figure 3. Flowchart of the speed sensor failure diagnosis algorithm

Furthermore, this proposed SSF diagnosis method can effectively apply for IMs' applications at the low-speed range where the speed sensor signal often suffers from noise while other similar algorithms would be complicated to handle in that such condition. That advantage comes from the idea of independently using the stator current model to detect the speed sensor fault because the current sensor signal is less affected by the noise in various speed zones. It means that any failure condition of the speed sensor can be 
clearly distinguished according to the difference between the real and estimated stator currents even though the noise affecting the speed sensor signal. Thus, the proposed approach can be effectively applied to diagnose the failure of speed sensors in various speed ranges.

\section{SIMULATION RESULTS}

In this part, the proposed SSF diagnosis method has been verified through various operation speeds of the IM in the case of totally disconnecting the speed sensor signal. The simulations have implemented in the MATLAB/SIMULINK environment with the parameters of the IM shown in Table 1.

Table 1. The parameters of the induction motor

\begin{tabular}{lccr}
\hline \multicolumn{1}{c}{ Description } & Symbol & Unit & Value \\
\hline Rated Power & $\mathrm{P}_{\mathrm{n}}$ & $\mathrm{kW}$ & 2.2 \\
Rated Voltage & $\mathrm{U}_{\mathrm{n}}$ & $\mathrm{V}$ & 400 \\
Rated Torque & $\mathrm{T}_{\mathrm{n}}$ & $\mathrm{Nm}$ & 14.8 \\
Rated speed & $\omega_{\mathrm{n}}$ & $\mathrm{rpm}$ & 1420 \\
Rated stator current & $\mathrm{I}_{\mathrm{S}}$ & $\mathrm{A}$ & 4.85 \\
Stator resistance & $\mathrm{R}_{\mathrm{S}}$ & $\Omega$ & 3.179 \\
Rotor resistance & $\mathrm{R}_{\mathrm{R}}$ & $\Omega$ & 2.118 \\
Mutual inductance & $\mathrm{L}_{\mathrm{m}}$ & $\mathrm{H}$ & 0.192 \\
Moment of inertia & $\mathrm{J}$ & $\mathrm{Kgm}^{\wedge} 2$ & 0.047 \\
Pole pair number & $\mathrm{p}$ & - & 2 \\
Stator inductance & $\mathrm{L}_{\mathrm{S}}$ & $\mathrm{H}$ & 0.209 \\
Rotor inductance & $\mathrm{L}_{\mathrm{R}}$ & $\mathrm{H}$ & 0.209 \\
Rated Rotor flux & $\Psi_{\mathrm{Sn}}$ & $\mathrm{Wb}$ & 0.757 \\
\hline
\end{tabular}

\subsection{Normal-speed range}

In the first case, the IM is operated at the normal speed range with a constant load torque of 5 N.m from $\mathrm{t}=0.5 \mathrm{sec}$. As shown in Figure 4 below, the reference speed steps up $50 \%$ of the rated speed $(710 \mathrm{rpm})$ at $\mathrm{t}=0.5 \mathrm{sec}$ and keeps to $\mathrm{t}=3 \mathrm{sec}$, then reduces according to a ramp line from $3.0 \mathrm{sec}$ to $3.5 \mathrm{sec}$ back to $25 \%$ of the rated value $(355 \mathrm{rpm})$. Suppose at $\mathrm{t}=2.0 \mathrm{sec}$, there is a fault of the speed sensor occurring, and the value of the speed signal is down to zero immediately. Figure 5 depicted the instability and incorrect operation of the IM system without the FTC module.

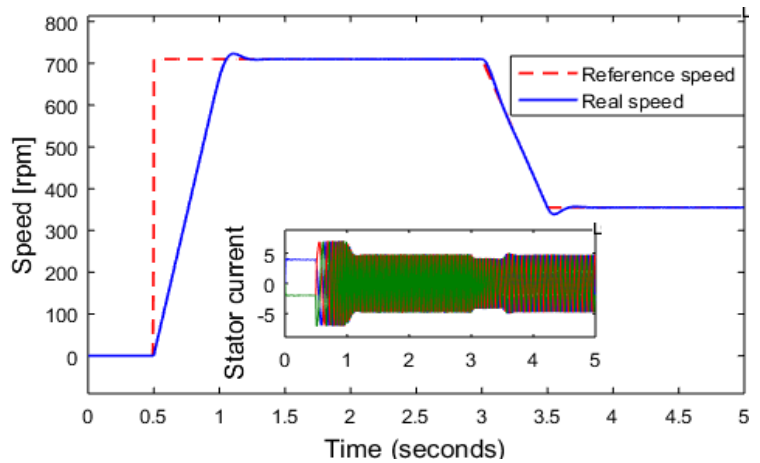

Figure 4. Rotor speed and three-phase current of IM in the healthy sensors-condition at normal-speed

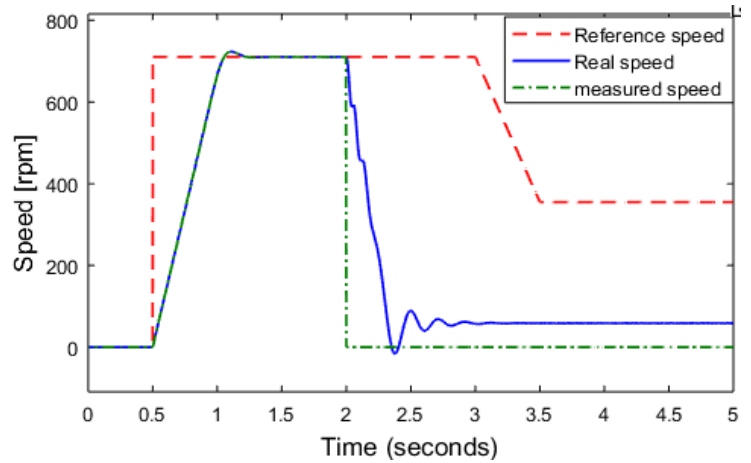

Figure 5. The rotor speed of IM in the SSF-condition at normal-speed without FTC method

Figure 6 shows the speed deviation between actual and estimated speed signals determined from the stator currents according to the $F_{\text {indication }}$ where it changes from zero corresponding to the normal condition to a significant value after $\mathrm{t}=2 \mathrm{sec}$ due to losing the sensor signal. According to (23), because this speed deviation is higher than Threshold 1-value, it makes the indication flag of the speed sensor failure going up immediately at that time. The performance of the proposed FTC method against the speed-sensor fault has been demonstrated in Figure 7. Here, when losing the feedback speed signal, the rotor oscillates in a short time, and then the FTC module quickly isolates the physical speed input and replaces it by the estimated rotor speed from the MRAS estimator to maintain the stable operation of the IM drive. The result shows that FTC implements the control action very well because the actual controlled speed keeps closely tracking the reference speed. 


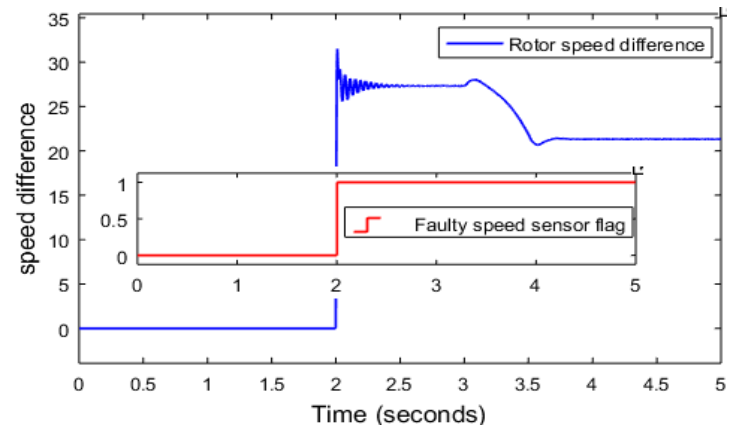

Figure 6. The rotor speed difference of diagnosis algorithm and the indication flag of SSF at normal-speed with the FTC method

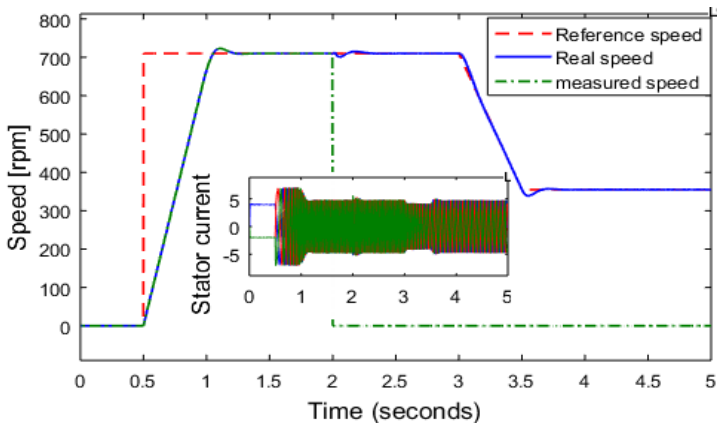

Figure 7. Rotor speed and three-phase current of IM in the SSF-condition at normal-speed with FTC method

\subsection{Low-speed range}

In this demonstration, we use the same control system and SSF diagnosis algorithm as the previous case. The reference speed steps up $10 \%$ of the rated speed $(142 \mathrm{rpm})$ at $\mathrm{t}=0.5 \mathrm{sec}$ and keeps to $\mathrm{t}=3 \mathrm{sec}$, and then reduces according to a ramp line from $3.0 \mathrm{sec}$ to $3.5 \mathrm{sec}$ back to $5 \%$ of the rated speed $(71 \mathrm{rpm})$. When occurring a speed sensor fault at $\mathrm{t}=2 \mathrm{sec}$, the sensor signal goes down to zero, and it makes the speed deviation calculated from the proposed SSF diagnosis algorithm changing rapidly at that time. Instantaneously, the FTC function is implemented against that such failure, and the indication flag surge to a high level, as shown in Figure 8. Then, the speed estimator will provide an alternative speed signal for the RFOC loop of the IM drive. As a result, the actual speed trajectory still perfectly keeps the speed reference and maintains a smooth operation at the low-speed range, even in the faulty sensor situation, as shown in Figure 9.

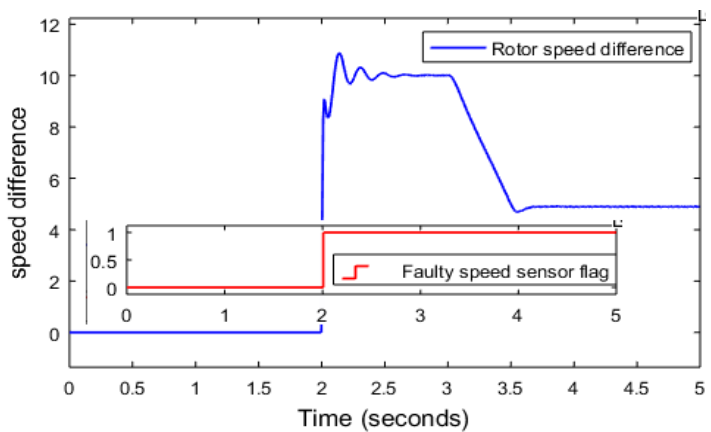

Figure 8. The rotor speed difference of diagnosis algorithm and the indication flag of SSF at low-speed with the FTC method

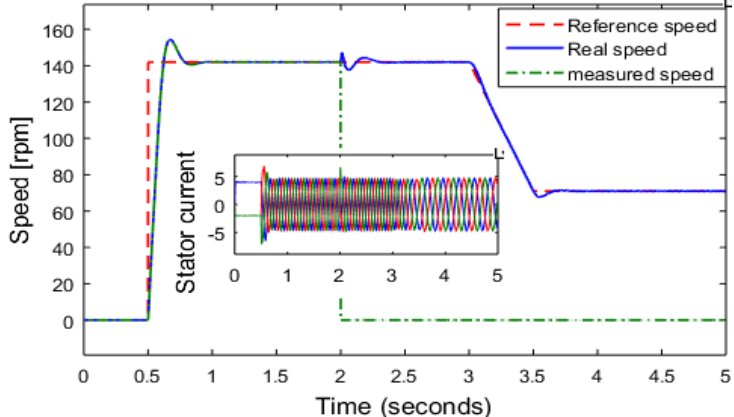

Figure 9. Rotor speed and three-phase current of IM in the SSF-condition at low-speed with FTC method

Furthermore, we have also implemented the traditional SSF diagnosis methods, which is based on the comparison between the measured and the estimated signals [10-12]. These simulations were performed by the parameters similar to the two cases above. Due to the simplicity of the diagnosis algorithm, the SSF-detection time of the traditional methods is shorter than the proposed method, and the faulty speed signal is quickly replaced by the estimated value. As a result, the speed characteristic applying the traditional diagnosis method achieves stability faster than that of using the proposed method, as shown in Figure 10. However, in practice, due to the noise affected on the measured speed signal in the low-speed operation, the traditional SSF diagnosis algorithm is not surely recognized the failure quickly as presented in the theoretical simulations.

Besides, a disadvantage of the traditional FTC methods is very sensitive to the estimated rotor speed, which could be affected by the quality of the speed estimation methods or current sensor failures. When occurring a current-sensor fault, the estimated speed value determined by the estimator is inaccurate; thus, it could be mistaken with the speed sensor failure and replace the healthy speed sensor signal by a wrong estimated value. Therefore, this misdiagnosis can lead to a breakdown of the whole IMD system. 
To verify the robustness and reliability of the proposed FTC method, some simulations under the confusional state with a current sensor failure have been implemented by both traditional and the proposed method. Here, the motor has been operated at normal and low-speed ranges while occurring failure of the current sensor in one phase. Figure 11 presents the performance of both traditional and proposed FTC methods at a reference of 50\% of the rated speed while occurring a current sensor failure in phase $\mathrm{A}$ at $\mathrm{t}=2.5 \mathrm{sec}$. As shown in Figure 11(b), the performance of the traditional FTC approach is unsuccessful from the moment of occurring the current sensor failure because the SSF diagnosis is mistaken, and its controller is switched to using the inaccurate estimated speed value. In contrast, the IM system operated under the proposed FTC method still keeps stably in the same situation because the SSF diagnosis has recognized exactly the trouble in the current sensor that is not the failure of the speed sensor. It is presented in Figure 11(c).

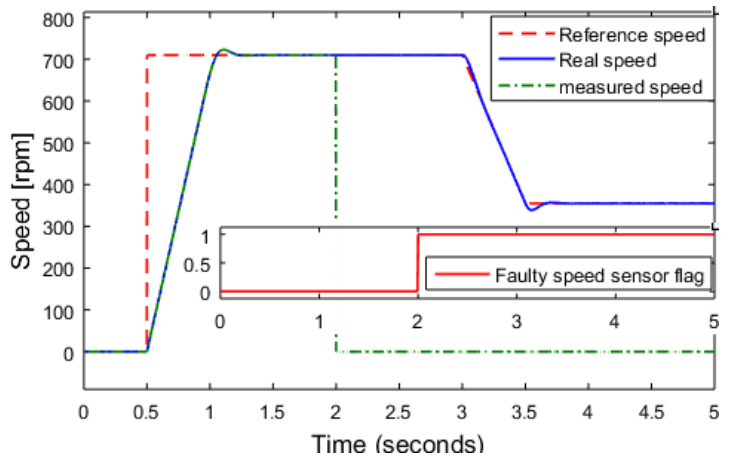

(a)

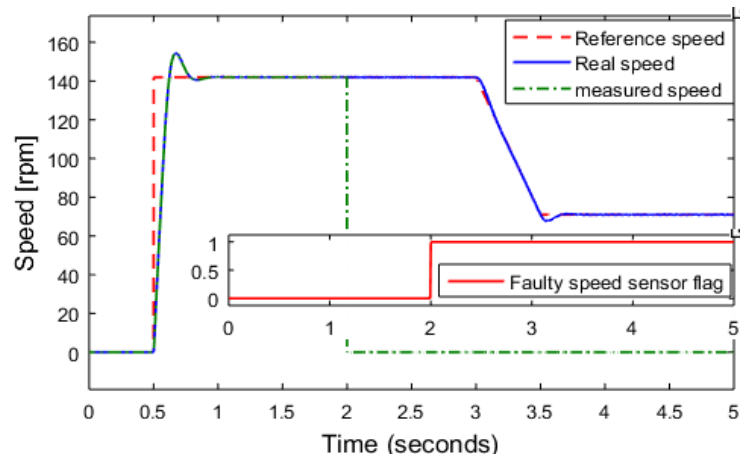

(b)

Figure 10. The rotor speed of IMD in the SSF-condition with traditional FTC method: (a) normal-speed range, (b) low-speed range

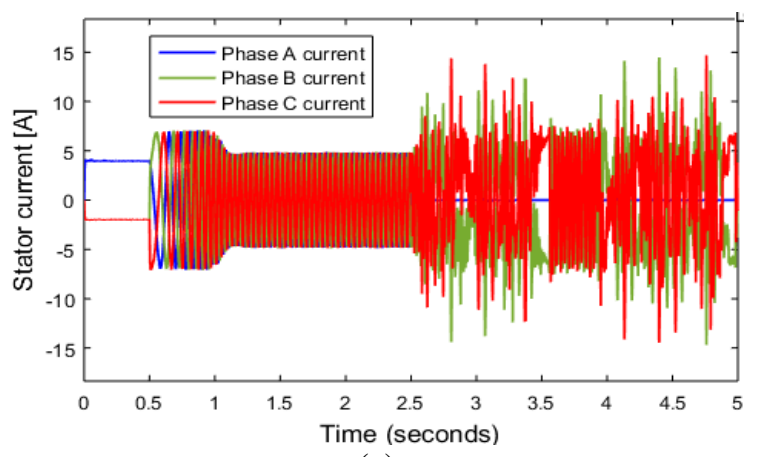

(a)

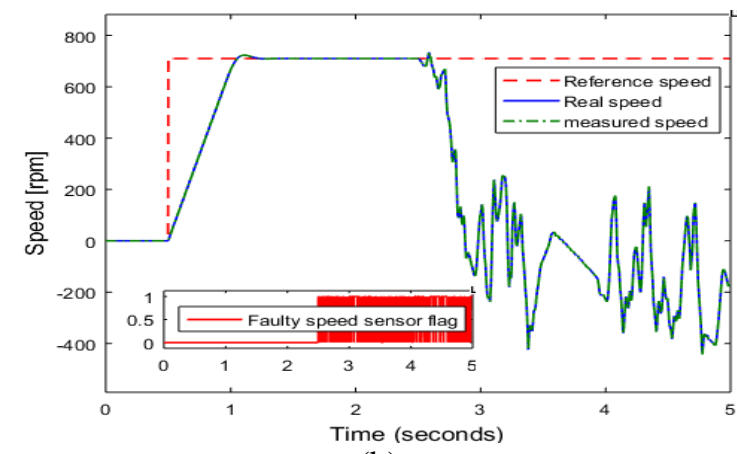

(b)

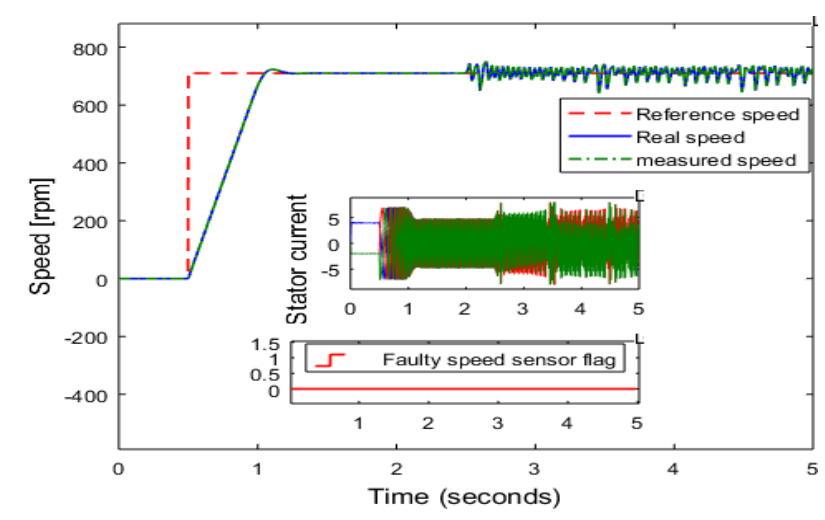

Figure 11. Comparison between traditional and proposed FTC methods at normal speed range:

(a) Three-phase stator currents (current failure in Phase A),

(b) Performance of the traditional FTC method with a current sensor failure,

(c) Performance of the proposed FTC method with a current sensor failure 
Similarly, Figure 12 presents the performance of both traditional and proposed FTC methods at a reference of $10 \%$ of the rated speed while occurring a current sensor failure in phase A at $\mathrm{t}=2.5 \mathrm{sec}$. As shown in Figure 12(b), the performance of the traditional FTC approach is totally unstable from the moment of occurring the current sensor failure because the SSF diagnosis is mistaken, and its controller is switched to using the inaccurate estimated speed value. In contrast, the proposed SSF diagnosis can recognize exactly the trouble in the current sensor, not the failure of the speed sensor. Still, due to the lack of the stator currents, the control loop of the FTC mode is unable to keep the appropriate performance of IMs even though it still is much better than the performance of traditional FTC methods. Figure 12(c) shows the operation of IDM based on the proposed FTC method in the low-speed range.

Therefore, from the results depicted by Figures 11 and 12, we can say that under a current sensor fault, the traditional FTC method makes the IDM system collapsed utterly. On the contrary, thanks to the preventive function against the mistake with the failure of the current sensor, the proposed SSF diagnosis approach works effectively. Thus, the idea of using the stator current mode in the proposed SSF diagnosis algorithm combined with the confusion prevention function has proved its effectiveness for IMs' applications in practice.

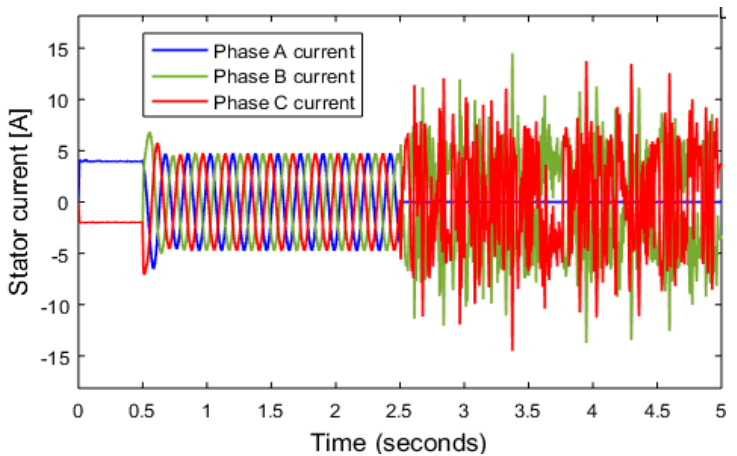

(a)

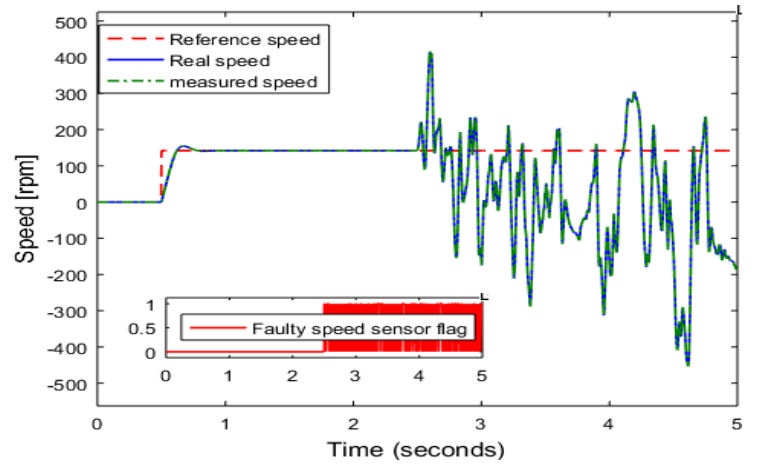

(b)

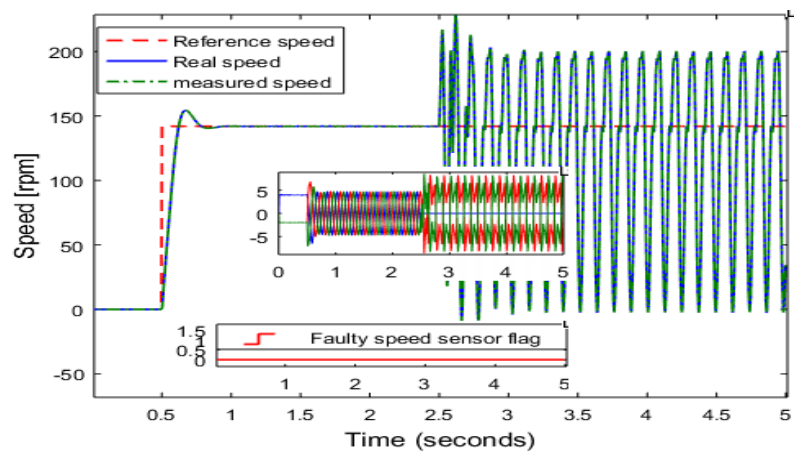

(c)

Figure 12. Comparison between traditional and proposed FTC method at the low-speed range:

(a) Three-phase stator currents (current failure in Phase A);

(b) Performance of traditional FTC method with a current sensor failure;

(c) Performance of proposed FTC method with a current sensor failur

\section{CONCLUSION}

This paper has proposed a novel diagnosis method based on the stator current model combined with the confusion prevention condition, which avoids mistaking the current sensor fault with the speed sensor fault. The advantage of the proposed algorithm is the separation between the diagnosis step and the reconfiguration step in the FTC mode of IMDs. A modified estimation algorithm of the RTC is also provided to strengthen the accuracy of the diagnosis method. Various tests have been performed to verify the effectiveness and robustness of the proposed approach in many speed ranges. The simulation results have proved the high competence of the proposed method to accurately detect the failure of the speed sensor and keep a stable operation of IM drive systems at various speed ranges. It could be a new approach to the diagnosis algorithm applied to detect multiple sensor failures. 


\section{ACKNOWLEDGEMENTS}

This work was supported by Project reg. no. SP2019/113 - Student Grant Competition of VSB-Technical University of Ostrava, 2019.

\section{REFERENCES}

[1] C. B. Regaya, et al., "A novel adaptive control method for induction motor based on Backstepping approach using dSpace DS 1104 control board," Mechanical Systems and Signal Processing, vol. 100, pp. 466-481, 2018.

[2] G. Kohlrusz and D. Fodor, "Comparison of scalar and vector control strategies of induction motors," Hungarian Journal of Industry and Chemistry, vol. 39, no. 2, pp. 265-270, 2011.

[3] T. O.-Kowalska, et al., "Fault-Diagnosis and Fault-Tolerant-Control in Industrial Processes and Electrical Drives," In Advanced Control of Electrical Drives and Power Electronic Converters, Springer, Cham, pp. 101-120, 2017.

[4] A. Lebaroud and G. Clerc, "Classification of induction machine faults by optimal time-frequency representations," IEEE Transactions on Industrial Electronics, vol. 55, no. 12, pp. 4290-4298, 2008.

[5] T. A. Najafabadi, et al., "Detection and isolation of speed-, DC-link voltage-, and current-sensor faults based on an adaptive observer in induction-motor drives," IEEE Transactions on Industrial Electronics, vol. 58, no. 5, pp. 1662-1672, 2010.

[6] K. Klimkowski and M. Dybkowski, "A fault tolerant control structure for an induction motor drive system," automatika, vol. 57, no. 3, pp. 638-647, 2016.

[7] L. Baghli, et al., "Sensor fault detection for fault tolerant vector controlled induction machine," In 2005 European Conference on Power Electronics and Applications, IEEE, pp. 10, 2005.

[8] A. A. Amin and K. M. Hasan, "A review of Fault Tolerant Control Systems: Advancements and applications," Measurement, 2019.

[9] A. A. Amin and K. M. Hasan, "Advanced Fault Tolerant Air-Fuel Ratio Control of Internal Combustion Gas Engine for Sensor and Actuator Faults," IEEE Access, vol. 7, pp. 17634-17643, 2019.

[10] F. Wu, et al., "A real-time sensor fault detection, isolation and reconfiguration method for vector controlled induction motors based on Extended Kalman Filter," In 2016 International Symposium on Power Electronics, Electrical Drives, Automation and Motion (SPEEDAM), IEEE, pp. 617-624, 2016.

[11] Y. Azzoug, et al., "Fault Tolerant Control for Speed Sensor Failure in Induction Motor Drive based on Direct Torque Control and Adaptive Stator Flux Observer," In 2018 International Conference on Applied and Theoretical Electricity (ICATE), IEEE, pp. 1-6, 2018.

[12] C. Chakraborty, V. Verma, "Speed and current sensor fault detection and isolation technique for induction motor drive using axes transformation," IEEE Transactions on Industrial Electronics, vol. 62, no. 3, pp. 1943-1954, 2014.

[13] K. Klimkowski and M. Dybkowski, "A comparative analysis of the chosen speed sensor faults detectors for induction motor drives," In 2015 International Conference on Electrical Drives and Power Electronics (EDPE), IEEE, pp. 333-338, 2015.

[14] F. Farhani, et al., "Real time PI-backstepping induction machine drive with efficiency optimization," ISA transactions, vol. 70, pp. 348-356, 2017.

[15] P. Brandstetter, "Sensorless Control of Induction Motor Using Modified MRAS," In International Review of Electrical Engineering (I.R.E.E), vol. 7, no. 3, pp. 4404-4411, 2012.

[16] M. S. Zaky, et al., "Review of different speed estimation schemes for sensorless induction motor drives," J Elect Eng, vol. 8, no. 2, pp. 102-40, 2008.

[17] S. M. Gadoue, et al., "Performance evaluation of a sensorless induction motor drive at very low and zero speed using a MRAS speed observer," In 2008 IEEE Region 10 and the Third international Conference on Industrial and Information Systems, IEEE, pp. 1-6, 2008.

[18] P. Brandstetter and M. Kuchar, "Rotor flux estimation using voltage model of induction motor," In 2015 16th International Scientific Conference on Electric Power Engineering (EPE), IEEE, pp. 246-250, 2015.

[19] C. D. Tran, et al., "Improving Fault Tolerant Control to the one current sensor failures for induction motor drives," In International Conference on Advanced Engineering Theory and Applications, Springer, Cham, pp. 789-798, 2018.

[20] S. A. Bednarz, et al., "Compensation of the Rotor Faults in the Vector Controlled Induction Motor Drive Using Parameter Estimator," In 2018 International Symposium on Electrical Machines (SME), IEEE, pp. 1-5, 2018.

[21] K. Klimkowski, et al., "Influence of stator and rotor resistances changes to the properties of the Fault Tolerant Vector Control of induction motor drive," In 2017 International Symposium on Electrical Machines (SME), IEEE, pp. 1-5, 2017.

[22] S. Bednarz, "Rotor fault compensation and detection in a sensorless induction motor drive," Power Electronics and Drives, vol. 2, no. 1, pp. 71-80, 2017.

[23] S. Gong and Z. Li, "Induction motor vector control based on adaptive identification of rotor resistance," In 2012 International Conference on Control Engineering and Communication Technology, IEEE, pp. 145-148, 2012.

[24] M. N. Gayathri, et al., "Performance of vector controlled induction motor drive with reactive power based MRAS rotor resistance estimator," In 2011 International Conference on Recent Advancements In Electrical, Electronics and Control Engineering, IEEE, pp. 352-356, 2011.

[25] P. Rokhforoz and J. Poshtan, "Rotor speed and resistance estimation using robust extended Kalman filter for sensorless vector control of induction motor drives," In The 6th Power Electronics, Drive Systems \& Technologies Conference (PEDSTC2015), IEEE, pp. 304-309, 2015. 